Trascender, Contabilidad y Gestión. Vol. 7, Núm. 19 (enero - abril del 2022).

Universidad de Sonora. Departamento de Contabilidad. México.

ISSN: 2448-6388. Reserva de Derechos 04-2015-04172070800-203.

\title{
Hacia una perspectiva integral de gestión en sostenibilidad empresarial
}

\section{Towards a comprehensive management perspective in business sustainability}

\author{
Magdalena Miranda Pegueros ${ }^{1}$; Elda Magdalena López Castro ${ }^{2}$; Cesar Vega Zarate ${ }^{3}$
}

Recibido: 8 de agosto de 2021.

Aceptado: 14 de octubre de 2021.

DOI: https://doi.org/10.36791/tcg.v7i19.136

JEL: Q56. Sostenibilidad.

M14. Responsabilidad social corporativa.

\section{Resumen}

La Agenda 2030 trae consigo una serie de objetivos encaminados al logro del desarrollo sostenible, dentro de estos se enmarca la importancia de las empresas para generar cambios en este rubro, ya que poseen la capacidad de transformar a su entorno utilizando la gestión estratégica como herramienta clave, la cual, para ser usada eficazmente, debe de conciliar tanto las necesidades de las empresas como de la sociedad. La presente investigación es de tipo descriptivo basado en fuentes documentales con un método deductivo de abordaje hermenéutico, en razón de que se efectuaron revisiones a las teorías de Responsabilidad Social Empresarial (RSE), Stakeholders y Sostenibilidad Empresarial como fuentes para el análisis de la gestión en la materia, las cuales, se sometieron a interpretación con el objetivo de llegar al planteamiento de su conceptualización integral que proporcione un bosquejo general de las partes involucradas, y donde se reconozca la divergencia entre las metas empresariales y las que la sociedad demanda, y al hacer esto trabajar en conjunto para reconciliar y abordar exitosamente a la sostenibilidad.

\footnotetext{
${ }^{1}$ Magdalena Miranda Pegueros. Maestra en Finanzas Corporativas. Investigadora independiente del programa de Doctorado en Ciencias Administrativas y Gestión para el Desarrollo en la Universidad Veracruzana, Xalapa, Veracruz, México. Correo: m4gd1t4@gmail.com. ORCID: 0000-0002-9028-6254.

2 Elda Magdalena López Castro. Doctora en Ciencias Administrativas y Gestión para el Desarrollo por la Universidad Veracruzana, Xalapa, Veracruz, México. Correo: eldlopez@uv.mx. ORCID: 0000-0002-3095-6682.

${ }^{3}$ Cesar Vega Zarate. Doctor en Ciencias Administrativas y Gestión para el Desarrollo por la Universidad Veracruzana, Xalapa, Veracruz, México. Correo: cevega@uv.mx. ORCID: 0000-0002-0002-4536.
}

Miranda Pegueros, M., López Castro, E. M., y Vega Zarate, $\mathrm{C}$.
Vol. 7, núm. 19 / enero - abril del 2022 DOI: https://doi.org/10.36791/tcg.v7i19.136 
Con esto se espera aportar bases teórico-conceptuales que permitan el desarrollo de futuras investigaciones.

Palabras claves: sostenibilidad, empresas, gestión, responsabilidad.

\section{Abstract}

The 2030 Agenda brings with it a series of objectives aimed at achieving sustainable development, within these is framed the importance of companies to generate changes in this area, since they have the ability to transform their environment using strategic management as a key tool, which, to be used effectively, must reconcile both the needs of companies and society. This research is descriptive based on documentary sources with a deductive method of hermeneutical approach, because revisions were made to the theories of Corporate Social Responsibility (CSR), Stakeholders and Business Sustainability as sources for the analysis of management in the matter, which were submitted to interpretation in order to arrive at the approach of its integral conceptualization that provides a general outline of the parties involved, and where the divergence between the business goals and those that society demands is recognized, and at the do this work together to reconcile and successfully address sustainability. With this, it is expected to provide theoreticalconceptual bases that allow the development of future research.

Keywords: sustainability, companies, management, responsibility.

\section{Introducción}

La sostenibilidad puede abordarse desde una perspectiva de equilibrio entre los aspectos económicos, sociales y ambientales de una sociedad, que permitan mantener la calidad de vida de sus futuras generaciones. Sin embargo, obtener en una balanza dichos aspectos y que ésta se mantenga calibrada ha sido una tarea que se ha trabajado a nivel internacional desde 1986 con el surgimiento del modelo del desarrollo sostenible propuesto por la Organización de las Naciones Unidas (ONU).

Los esfuerzos para lograr la sostenibilidad se han reflejado en la Agenda 2030 para el Desarrollo Sostenible, cuyas medidas de acción de los países miembros, abarcan una serie de estrategias encaminadas a cumplir 17 objetivos propuestos, a través de soluciones multidisciplinarias que aborden a los actores involucrados. Aquí se reconoce a la empresa como parte fundamental de la economía y, por lo tanto, tiene un papel clave para la integración de la sostenibilidad, esto se encuentra definido en el objetivo 12.6 el cual aborda la importancia de la gestión empresarial para la generación de informes en la materia que faciliten la comunicación con los grupos de interés (ONU, 2015).

En ese sentido, la gestión empresarial se convierte en una herramienta para incorporar la sostenibilidad, por lo que se busca a través de abordajes teóricos una aproximación que permita obtener resultados en la materia. Tal es el caso de la teoría de la responsabilidad social empresarial (RSE), que ha sido debatida extensamente desde sus orígenes formales a mediados de los años 50’s en diferentes ámbitos académicos y empresariales, actualmente se encuentra ampliamente aceptada y estandarizada a través de normas como lo son la RC/EFQM, SA8000, EFR1000 e ISO26000.

Sin embargo, a pesar de los esfuerzos aún no se obtienen los resultados esperados, toda vez que, la concepción de la RSE ha dejado de lado la naturaleza misma de la empresa, cuya motivación nace del objetivo 
de crear utilidades para sus accionistas, distando mucho de la naturaleza voluntaria que propone la RSE, por lo que, en pos del logro de una gestión encaminada a la sostenibilidad, se deben de conciliar ambos objetivos.

A lo largo del presente trabajo se desarrollará la construcción de una conceptualización integral de gestión en sostenibilidad empresarial, a través del análisis descriptivo basado en fuentes documentales con un método deductivo de abordaje hermenéutico, abordándola desde las teorías de RSE, Stakeholders y Sostenibilidad empresarial. Lo anterior permitirá la extracción de premisas que permitan una construcción teórico conceptual que sirva de base para futuras investigaciones en la materia.

\section{Desarrollo}

\section{Responsabilidad Social Empresarial (RSE)}

La RSE ha sido una corriente teórica que ha intentado analizar los efectos que la empresa tiene en su entorno, convirtiéndola en materia de estudio de las ciencias administrativas desde una perspectiva de la gestión de dichos impactos. Aunque, hablar de la RSE como la simple gestión de externalidades quedaría muy corto, ya que sus alcances se reflejan en diversas áreas dentro de la organización, por lo tanto, se trata de una teoría transversal que se encuentra en constante evolución (Crane et al., 2009; Carroll, 2015).

Los orígenes de la RSE se pueden situar en una época anterior a la década de los años 1950 (época en la cual teóricamente se ubica esta corriente), ya que se ha encontrado evidencia de que empresas ubicadas en el siglo XIX presentaban comportamientos dirigidos a un mejoramiento en la calidad de vida de su entorno, aunque este comportamiento se relacionaba directamente con los ideales de los propietarios y no por el reconocimiento de las afectaciones que estas generaban (Carroll, 2009).

Una de las primeras muestras de esta concientización formal se ubica en 1946 en una encuesta realizada por la revista Fortune a los empresarios sobre su responsabilidad social en la cual "el 93.5\% reconocía esas responsabilidades y hacia lo mejor para satisfacerlas, así mismo, se mostraba que el porcentaje de la empresa que se enfocaba a este rubro variaba entre un cuarto y la mitad de la empresa” (Fortune, 1946 citado por Carroll, 2009, p. 7).

A este periodo, Carroll (2009) lo describe como aquel que sentó las bases para la formalización de la RSE, ya que se logra su conceptualización teórica por parte de Bowen (2013) quien la define como “aquellas obligaciones de los empresarios de seguir políticas, tomar decisiones o seguir líneas de acción que son deseables en términos de los objetivos y valores de nuestra sociedad” (p.6).

En este sentido, el uso del término pasa al lenguaje corporativo y su transformación se encamina a la gestión de la participación de la empresa tanto en problemas sociales como ambientales, para mitigar el impacto negativo a su entorno, o incluso lograr generar resultados positivos (Husted y Salazar, 2005).

Su legitimación a través de normas como la ISO 26000, proporciona un consenso en la metodología dentro de la gestión empresarial para alcanzar una responsabilidad social, pero sobre todo para alcanzar un 
desarrollo sostenible. Esta afirmación da lugar a un análisis de la responsabilidad social en las tres aristas que maneja el desarrollo sostenible ${ }^{1}$, las cuales son: económica, medio ambiental y social (Morales-Castro, 2015).

El reconocimiento del impacto de las empresas a su entorno y el consenso de que la RSE refleja el comportamiento ideal para las empresas son elementos que se pueden encontrar dentro de la conceptualización de la teoría, por lo tanto, su importancia radica en la premisa de que la empresa es un ente capaz de transformar a su entorno, y a través de una gestión sostenible promueve beneficios que se expanden más allá de su marco de operación.

Mientras que, por otro lado, la motivación que lleva a las empresas a incorporar este comportamiento ha sido motivo de controversias y depende de la perspectiva del autor o de organismos internacionales, tal es el caso de la Unión Europea, que considera a la RSE como aquella gestión, que independientemente de las obligaciones de carácter legal, la empresa asume voluntariamente en materia ambiental o social (Comisión Europea, 2011). De ahí que, la naturaleza voluntaria de esta conceptualización genera críticas encaminadas a una percepción que argumenta que, si la motivación se encontrara encaminada en esta dirección no se tendría la necesidad de legislar el buen comportamiento empresarial (Blowfield y Frynas, 2005).

Salazar y Husted (2009) identifican que la empresa puede tener tres tipos diferentes de motivación que generen resultados en materia de una gestión en sostenibilidad, la primera considera una naturaleza voluntaria, como es el caso de la dirección bajo la cual se aborda la RSE; la segunda parte de una perspectiva coercitiva en donde se involucra una regulación de ciertos aspectos que las empresas se ven obligadas a cumplir para evitar incurrir en sanciones; y la última, se encarga de la visión estratégica de la empresa, reconociendo tanto la búsqueda del beneficio social como la obtención de beneficios económicos.

Con el abordaje de Salazar y Husted (2009), se puede concluir que no se encuentran elementos suficientes para afirmar que la motivación de tipo voluntario genere incentivos empresariales fuertes para integrar un cambio en el comportamiento empresarial, con esta premisa se abre la pauta para realizar a continuación un análisis a fondo de las motivaciones coercitivas y estratégicas.

\section{Los Stakeholders como promotores del cambio empresarial}

La sociedad misma no se queda a la espera de la buena voluntad de las empresas y de sus accionistas, y busca a través de la coerción ejercer presión para lograr algún cambio. En este sentido, la teoría de lo Stakeholders que fue propuesta por Freeman (2010), considera a los grupos de interés como aquellos grupos o individuos que se benefician o perjudican por las acciones corporativas tales como clientes, proveedores, sociedad, gobierno, ONG's, etc.

Esta teoría se basa en la toma de decisiones organizacionales encaminadas no sólo a la satisfacción de los accionistas sino a la responsabilidad que tienen hacia sus grupos de interés para satisfacer sus

\footnotetext{
${ }^{1}$ La sostenibilidad se entiende como el fin, mientras que la RSE es la parte teórica que ayuda a comprender el proceso para su logro desde la perspectiva empresarial. La evolución teórica de la RSE ha alcanzado un punto en que su internalización empresarial converge en el logro de los Objetivos del Desarrollo Sostenible (ODS) propuestos en la Agenda 2030. Teniendo relevancia en la Teoría de la RSE para promover el replanteamiento e inclusión de diversos aspectos sociales como lo son: logros en la equidad y justicia, ética y derechos humanos que originalmente no se consideraban en la conceptualización de la sostenibilidad (Plasencia et al., 2018).
} 
necesidades. Bajo esta óptica, se podría considerar que la RSE entra bajo una perspectiva de un compromiso empresarial hacia la sociedad y sus peticiones, como señala Melé (2009): "Si tomamos la RSE en un sentido amplio, entonces la teoría de los Stakeholders puede considerarse una teoría de la RSE, ya que proporciona un marco normativo para los negocios responsables hacia la sociedad” (p. 63).

Bajo esta óptica los grupos de interés son tomados en cuenta como socios de la empresa, a los cuales se les debe de considerar y maximizar la obtención de beneficios sociales ${ }^{1}$. Por lo que, la gestión empresarial debe dirigirse no solo a la búsqueda de utilidades para los accionistas sino también para los grupos de interés, con el fin de que las demandas que estos les imponen surtan efectos a través de los marcos normativos vigentes.

A través de esta teoría, se reconoce la premisa de que los grupos de interés son parte fundamental del sistema en el que se desarrolla la empresa ya que pueden afectar a la toma de decisiones empresariales (Agle et al., 2008; Fernández y Bajo, 2012; Freeman, 2010), esto mediante la presión que estos grupos pueden ejercer para demandar bienestar, y generar un cambio en las legislaciones que puedan repercutir en multas o sanciones.

En esta misma línea, autores como Agle et al. (2008) consideran al gobierno como elemento indispensable, ya que se encarga de establecer las reglas del juego a través de la promulgación de leyes, es decir, modelar el comportamiento de las empresas que se encuentran dentro del sistema; para lograr la maximización del valor total a largo plazo de la empresa. Por lo que, se acepta la premisa de que el gobierno es el único encargado de la resolución de los problemas, pero bajo su papel de gestor de las reglas del juego (promulgación de leyes) y como vigilante, con el fin de minimizar los costos impuestos a la sociedad derivados de un comportamiento empresarial desmedido en materia de sostenibilidad.

En virtud de lo anterior, se entiende que esta problemática no puede ser resuelta a nivel micro, es decir, entre empresas e individuos y, por tanto, la búsqueda de soluciones efectivas debe de originarse mediante acciones colectivas de los grupos de interés, planteando una perspectiva en donde la coerción va moldeando el comportamiento de la empresa a través de leyes promulgadas.

La coacción de los grupos de interés permite identificar que la motivación empresarial puede ser modificada y pasar de ser voluntaria a obligatoria (siempre y cuando exista una coordinación que genere sincronía en los procesos), sin embargo, para ser efectiva se requiere una presencia fuerte de organismos vigilantes que validen el cumplimiento de las leyes.

Para esto, un flujo adecuado de la información entre gobierno, grupos de interés y empresas sienta las bases para un correcto proceso en la toma de decisiones. Lo anterior ayuda a evitar el surgimiento de algún tipo de conflicto y de esta forma lograr un equilibrio entre los múltiples intereses que puedan surgir (Narayanan y Adams, 2017). Por lo que, se acepta la siguiente premisa: el flujo de información juega un papel importante para la reducción de los costes de transacción, que genera una sinergia entre los Stakeholders y la empresa, esta última construye una imagen a través de esto.

\footnotetext{
${ }^{1}$ Los beneficios sociales se entienden como aquellos que responden a las demandas de sostenibilidad, y en este trabajo se encaminan a los establecidos en los ODS enlistados en la Agenda 2030.
}

Miranda Pegueros, M., López Castro, E. M., y Vega Zarate, C.
Vol. 7, núm. 19 / enero - abril del 2022 DOI: https://doi.org/10.36791/tcg.v7i19.136

Pp. 150-164 
Kuhn y Deetz (2009) hacen énfasis en el establecimiento de canales de comunicación que logren establecer un círculo participativo de continua interacción de las partes interesadas (tanto accionistas como grupos de interés), que operen dentro de un proceso que implique la resolución de conflictos de forma creativa, para el logro de sus objetivos.

La necesidad de un estándar en el flujo de información de las actividades empresariales así como de su divulgación, se ha reconocido desde la década de los 1980 en las prácticas de la Comisión de Bolsa de Valores en los Estados Unidos de América, lugar en donde ha pasado a formar parte de la estandarización de la presentación de información que facilite su vigilancia y evitar problemas de comunicación entre los grupos de interés y las empresas (Owen y O’Dwyer, 2009).

La metodología aplicada en estos tipos de balances sociales debe incluir aquellos beneficios adicionales a los económicos que las organizaciones transfieren a sus grupos de interés, por lo que, Molina, et al. (2017) definen al balance social como: "un sistema de diagnóstico que integra evaluaciones cuantitativas a través de indicadores sociales, ambientales y financieros; así como cualitativas sobre cumplimiento de normas y principios sociales” (p. 25).

En este sentido, la estandarización relativa a la sostenibilidad para evitar cualquier tipo de conflicto, ha sido impulsada por el Programa de Medio Ambiente de las Naciones Unidas (PNUMA) y la Coalition for Environmentally Responsible Economies denominada Reporting Global Initiative (GRI) que actualmente es el punto de referencia en materia de generación de reportes empresariales en sostenibilidad que respetan los principios del Triple Botton Line ${ }^{1}$.

Narayanan y Adams (2017) afirman que el proceso de presentación de informes sobre cuestiones de sostenibilidad tiene el potencial de cambiar a las organizaciones, solo sí el enfoque con el que se adopta, respeta la búsqueda del lucro organización y las prácticas de cálculo asociadas; y si bien, bajo este enfoque se comprobó que se limita la profundidad del cambio, se demostró la existencia de cierto grado de integración, por lo que sí la búsqueda se enfoca en la integración de sostenibilidad entonces se debe analizar y detectar qué funciona y qué no en términos de generar un cambio intraorganizacional.

\section{La sostenibilidad empresarial y la divergencia de los objetivos sociales y económicos}

El análisis de los grupos de interés proporciona elementos esenciales para la construcción de una conceptualización integral de gestión en sostenibilidad empresarial, ésta aún carece de un elemento esencial que muchas veces se deja de lado cuando se demanda de las empresas un comportamiento sostenible: las utilidades.

El impacto económico que se presenta dentro de las empresas cuando se generan acciones en materia de RSE, no ha sido un elemento identificable dentro de la conceptualización general del término; en este sentido, la empresa es un ente económico que lucha constantemente para mantener un equilibrio entre la maximización de sus utilidades y las presiones que se ejercen sobre ella para la atención a temas de diferente índole social.

La crítica abordada por Friedman (2007), ha evidenciado principalmente esta dicotomía, basándose

\footnotetext{
1John Elkington acuñó la frase Triple Bottom Line que hace referencia a tres pilares diferentes: social, económico y ambiental, y el cual alienta a las empresas a ampliar su definición de éxito siendo incluyentes en sus estrategias de estos elementos (Rotner, 2016).
} 
en la Teoría de la Agencia que reconoce a dos partes involucradas: al principal (Stockholders o accionista) como fuente de autoridad; y al agente al cual se delega la misma para actuar en su nombre y solucionar los problemas que se puedan encontrar para hacer esto posible (Salazar y Husted, 2009).

Friedman (2007) considera la premisa de que el fin único de la empresa es obtener beneficios económicos para los accionistas. En el caso de los proyectos de RSE, en donde se busca generar un bienestar al entorno en el que se desenvuelve la empresa, la cual va en contra de este principio, abre la pauta para analizar la motivación que podría llevar a la su incorporación dentro de la gestión empresarial.

Por lo que, la revisión de la literatura enfocada a las motivaciones empresariales para la integración del cuidado de aspectos sociales como parte fundamental de sus actividades, se basa en la premisa de que: la estrategia es un mecanismo que genera una sinergia positiva tanto para la sociedad como para la empresa. Salazar y Husted (2009), reconocen una relación positiva entre los gastos y el desempeño competitivo y financiero de la empresa, que busque un equilibrio entre el beneficio social y el beneficio económico propio.

La motivación estratégica busca una constante legitimación y supervivencia en el mercado, para esto, se debe reconocer la importancia que adquiere la información que proyectan y la imagen que se construye alrededor de esta; las empresas que lo asuman podrán aprovechar y encontrar así, el camino para modificar su comportamiento y generar ventajas competitivas en el mercado, mientras que aquellas que no lo hacen pueden enfrentarse a un escenario negativo derivado de la perdida de potenciales clientes que buscan productos o servicios regidos bajo los conceptos de sostenibilidad (Adams et al., 2016; Ergene et al., 2020).

Miranda Pegueros, M., López Castro, E. M., y Vega Zarate, C.
Otro aspecto importante que se debe analizar es el impacto financiero de integrar a la sostenibilidad dentro de la gestión empresarial, este podrá ser medido por indicadores que permitan cuantificar su efecto (Gao y Bansal, 2013; Eccles et al., 2014; Earnhart, et al., 2014), aunque la importancia de estos será relevante en la medida en que ayuda a construir una imagen a través de sus reportes de información financiera, ayudando a la creación de una comunicación no verbal entre la empresa y sus grupos de interés (Adams y Larrinaga, 2007; Bebbington et al., 2008; Miranda y López, 2020).

Por lo anterior, se concluye con la premisa que aquellas empresas que utilicen una perspectiva estratégica en su actuar y como consecuencia generen beneficios sociales, mantendrán un equilibrio en la búsqueda de un desempeño financiero en la empresa (Candelas, 2017; Savitz y Weber, 2007); convirtiendo a las actividades de sostenibilidad como parte de una gestión para generar riqueza a los accionistas.

Sin embargo, la sostenibilidad no se trata solo de generar reportes que satisfagan a los grupos de interés; sino que realmente la empresa funja como un ente renovador de la sociedad proporcionando tanto un retorno económico como de beneficio social. La propuesta de Savitz y Weber (2007) visualiza esto, como un terreno común donde se intercambian beneficios entre los grupos de interés y la empresa; esta nueva forma de concebir la interacción reconoce que debe de existir un punto de equilibrio que satisfaga a ambas partes, a este lo denomina como el lugar dulce sostenible (The Sustainability Sweet Spot); que es donde confluyen las ganancias y la búsqueda del bien común, dando lugar y oportunidad para la generación de nuevos productos, procesos, mercados, modelos de negocios, métodos de gestión y reporteo.

La perspectiva estratégica para la empresa como guía
Vol. 7, núm. 19 / enero - abril del 2022
DOI: https://doi.org/10.36791/tcg.v7i19.136

Pp. 150-164 
para alcanzar ventajas competitivas a través de la productividad y de la optimización de los recursos, ayuda a la empresa a encontrar nuevas formas de hacer las cosas en comparación de su competencia y así generar algún tipo de valor agregado a través de la implementación de nuevos procesos en sostenibilidad (Adams, et al., 2016; Ergene et al., 2020).

\section{Aspectos metodológicos}

La presente investigación parte de un enfoque cualitativo, con un método deductivo de abordaje hermenéutico, ya que se efectuaron revisiones de contenidos generales sobre las teorías de RSE, Stakeholders y Sostenibilidad Empresarial, que se someten a interpretación para llegar al planteamiento conceptual integral de la gestión en sostenibilidad empresarial donde se reconcilien tanto los objetivos económicos como los sociales.

\section{Hacia una perspectiva integral de sostenibilidad}

Los estudios sobre las estrategias empresariales analizados anteriormente, se han enfocado en el análisis de elementos aislados, ya sea convirtiendo la coercitividad de las leyes en motivadores estratégicos(Lyon y Maxwell, 2007); considerando a la información financiera como la perfecta estrategia en búsqueda de una imagen sustentable (Adams y Larrinaga, 2007; Bebbington et al., 2008); o a través de la interpretación de la interacción entre la empresa y sociedad como una balanza en donde influyen fuerzas que deben de encontrar un punto de equilibrio para que ambos agentes ganen (Savitz y Weber, 2007), así como el reconocimiento de una oportunidad de un mercado cada vez más interesado en consumir productos sostenibles y por lo tanto especializarse en estos otorgará ventajas competitivas sobre aquellos que no lo hagan (Ergene et al., 2020; Adams, et al., 2016).

Sin embargo, a pesar de que la sostenibilidad puede ser abordada bajo diferentes ópticas, se deben de integrar elementos que sirvan como punto de partida a las nuevas teorías organizacionales, en pos de generar un conocimiento que ayude a construir mejores modelos representativos de la realidad que se analiza.

En términos conceptuales, se debe construir una visión integradora que se acepte la existencia de contradicciones, tensiones y paradojas ${ }^{1}$. Esta deberá de aceptar las tensiones en vez de eliminar o ignorar la problemática presente, por lo que, la capacidad de la dirección para perseguir aspectos de sostenibilidad, aparentemente conflictivos simultáneamente, logrará una conceptualización integral (Hahn et al., 2015).

El reconocimiento de la naturaleza económica de las empresas y su búsqueda permanente de rendimientos sobre cualquier otro fin, permite volver visible algo que siempre ha estado allí, pero que, raramente se incorpora a las teorías clásicas tanto de la RSE como de la Sostenibilidad Empresarial. Es solo a través de lo anterior expuesto que se ha afianzado en los últimos años esta corriente teórica que busca analizar la falta de éxito de la integración de lo sostenible a las corporaciones.

Por lo que, el debate en la Sostenibilidad Empresarial según Dyllick y Muff (2016), se encuentra bajo tres desafíos conceptuales que abordan la existencia de una desconexión: el primero hace énfasis en la pobre integración de las diferentes corrientes temáticas en este

\footnotetext{
${ }^{1}$ Una paradoja se refiere a una situación en la que coexisten elementos de oposición, porque hay dos o más elementos que son sólidos y aceptados individualmente ", pero tomados en conjunto parecen ser inconsistente o incompatibles (Hahn et al., 2015).
} 
discurso, el segundo aborda la falta de integración de la sociedad nivel macro con el nivel micro organizacional, y la tercera critica la construcción de la idea del éxito basado en obtención de grandes márgenes de utilidades, proponiendo abrir la idea de la obtención de un bien común como un logro igual de importante.

Si bien, el centro del debate parte de la relación entre los objetivos económicos, ambientales y sociales de la empresa, este se encuentra madurando hacia un enfoque más estrecho, que busca focalizar algunas preguntas clave y un conjunto limitado de lentes teóricos y enfoques metodológicos para abordarlas (Hang et al., 2018).

Otro desafío se refiere a la dificultad de medir el impacto social, a diferencia del valor económico, que puede medirse utilizando unidades monetarias específicas, el valor social no se puede cuantificar tan fácilmente. Lo que conlleva complicaciones en los aspectos metodológicos, ya que a menudo es difícil idear la forma correcta de medir la afección o falta de ella en un medio social. Además, no existe un tipo de cambio comúnmente aceptado para comparar el valor social y el valor económico. Por lo tanto, los gerentes deben decidir intuitivamente entre maximizar la seguridad financiera de una organización o aumentar su impacto social. Si la organización está considerando una inversión de las ganancias actuales para expandir el valor social futuro, esta decisión puede volverse aún más complicada (Delmas et al., 2019).

A través, de la revisión teórica realizada en el presente trabajo se identificaron ideas clave en las teorías revisadas, que permiten la construcción teórico conceptual de la gestión en sostenibilidad empresarial bajo una perspectiva incluyente, por lo tanto, se propone una nueva comprensión de la realidad basada en las siguientes premisas:
- Premisa 1: La empresa es un ente capaz de transformar a su entorno, y a través de una gestión sostenible promueve beneficios que se expanden más allá de su marco de operación.

- Premisa 2: No se encuentran elementos suficientes para afirmar que la motivación de tipo voluntario genere incentivos empresariales fuertes para integrar un cambio en el comportamiento empresarial.

- Premisa 3: Los grupos de interés son parte fundamental del sistema en el que se desarrolla la empresa ya que pueden afectar a la toma de decisiones empresariales mediante la presión que estos ejercen para demandar bienestar, y generar un cambio en las legislaciones.

- Premisa 4: El gobierno es el único encargado de la resolución de los problemas, pero bajo su papel de gestor de las reglas del juego (promulgación de leyes) y como vigilante, con el fin de minimizar los costos impuestos a la sociedad derivados de un comportamiento empresarial desmedido en materia de sostenibilidad.

- Premisa 5: El flujo de información juega un papel importante para la reducción de los costes de transacción, que genera una sinergia entre los Stakeholders y la empresa, esta última construye una imagen a través de esto

- Premisa 6: El fin único de la empresa es obtener beneficios económicos para los accionistas.

- Premisa 7: La estrategia es un mecanismo que genera una sinergia positiva tanto para la sociedad como para la empresa.
Miranda Pegueros, M., López Castro, E. M., y Vega Zarate, $\mathrm{C}$.
Vol. 7, núm. 19 / enero - abril del 2022 DOI: https://doi.org/10.36791/tcg.v7i19.136

Pp. 150-164 
- Premisa 8: Aquellas empresas que utilicen una perspectiva estratégica en su actuar y como consecuencia generen beneficios sociales, mantendrán un equilibrio en la búsqueda de un desempeño financiero en la empresa.

Con estas premisas podemos definir integralmente a la gestión en sostenibilidad empresarial como: aquella que se encuentra en constante interacción entre los integrantes del sistema (empresa, grupos de interés y gobierno) con el fin de obtener un equilibrio entre los diferentes objetivos que se buscan alcanzar (beneficio económico y beneficio social); esta interacción, genera un punto de equilibrio que proporciona un retorno del beneficio social óptimo al exterior como consecuencia de la presión hacia la empresa, y, un retorno del beneficio económico óptimo como consecuencia de las estrategias empresariales. Por lo que, la gestión sostenible buscará conciliar la obtención del mayor beneficio económico considerando las demandas sociales, esto a través de una perspectiva estratégica como se observa en la Figura 1.

\section{Figura 1}

Representación de la conceptualización integral de la Gestión en Sostenibilidad Empresarial

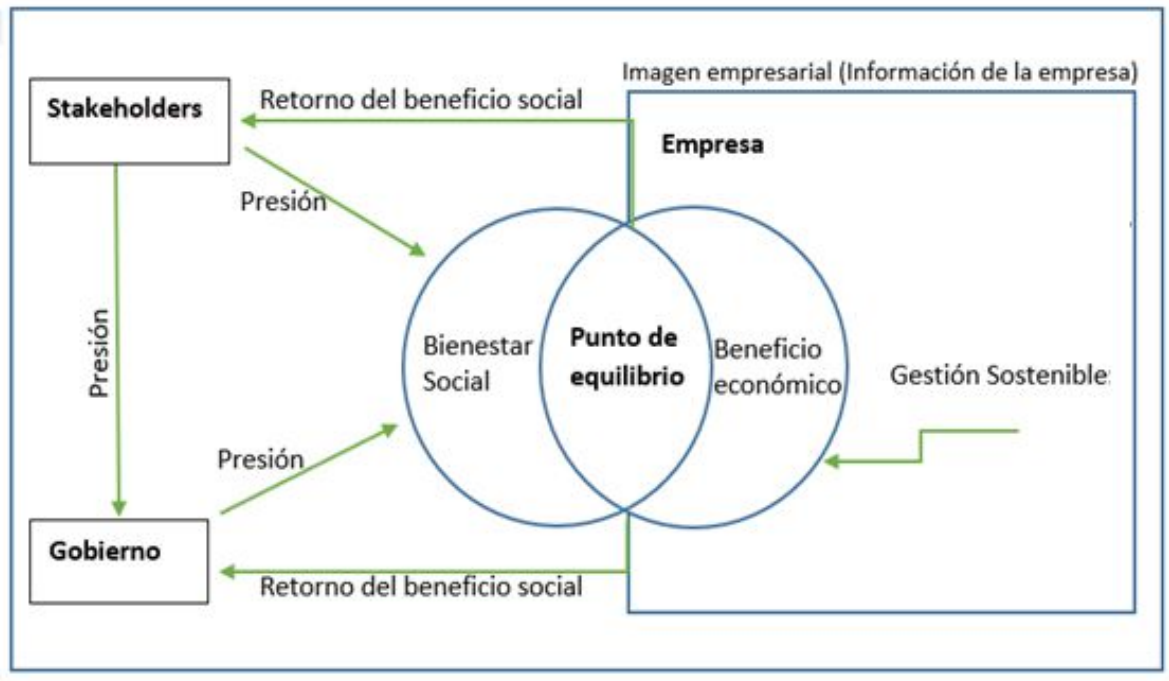

Fuente: Elaboración propia con base en Andrés et al. (2010) y Savitz y Weber (2007).

\section{Discusión}

La construcción conceptual abre la pauta para el debate sobre los aspectos analizados, por un lado, las empresas utilizan las prácticas de transparencia y de disponibilidad de información en sus reportes como vía para volverse sostenibles. Carroll (2015) indica que alrededor del 95\% de las empresas que aparecen en The Global Fortune 250 (GF250), revelan datos de forma voluntaria sobre aspectos de desempeño social, económico y ambiental. Mientras que, en regiones como América Latina el porcentaje es mucho menor, siendo Brasil el país donde las empresas generan más estos 
tipos de informes con una participación del 45\%, mientras que en México el porcentaje oscila entre el 10\% (Molina et al., 2017).

En México, la información en la materia se concentra en aquellas empresas públicas que se encuentran cotizando dentro de la Bolsa Mexicana de Valores (BMV), ya que es una institución que proporciona la infraestructura, la supervisión y los servicios en materia de emisión, colocación e intercambio de valores, y funge como órgano vigilante sobre las actividades de las empresas emisoras y casas de Bolsa.

La sostenibilidad en la BMV, busca contribuir al fortalecimiento de las prácticas de gestión y reporteo de sus empresas emisoras, con la finalidad de ofrecer información de calidad, relevante y transparente sobre aspectos ambientales, sociales y de gobernanza corporativa (ASG) a inversionistas, accionistas y grupos de interés; con el fin de proporcionar un ambiente óptimo para la toma de decisiones, en este marco pueden ser estudiados los aspectos de gestión sostenible a través de los reportes generados por las empresas así como el del comportamiento de las utilidades empresariales a través de sus índices de cotización.

Los indicen bursátiles también han incorporado aspectos de sostenibilidad, y a partir de diciembre del
2011 la BMV introdujo el IPC sostenible; el cual permite agrupar a empresas cuyo desempeño ambiental, social y de gobierno corporativo sean sobresalientes, con el fin de acceder al capital de inversionistas preocupados por dichos aspectos, además de que se identifican menores riesgos (Mayo et al.,2018).

Sin embargo, a partir de junio del 2020, este indicador desaparece para dar paso al S\&P/BMV Total México ESG Index, el cual actualmente se encuentra compuesto por 29 empresas. Esta transformación responde a la inclusión de elementos sobre ESG (por sus siglas en inglés: Environmental, Social y Governance), favoreciendo la inclusión dentro de las empresas mexicanas de dichos aspectos, a su vez que se encuentra respaldado por una alianza con el índice americano Standard \& Poor's (S\&P).

Lo anterior comprueba una creciente tendencia por parte de los inversionistas hacia los temas sostenibles, ya que la percepción de que una compañía que se gestiona en esta materia genera ventajas a largo plazo derivado de una mejor capacidad de adaptación (Pérez, 2018). Y esto puede comprobarse cuando se comparan los rendimientos promedios entre el IPC y el S\&P/BMV Total México ESG Index, en el cual se observa efectivamente la obtención de un mayor valor (Tabla 1).

\section{Tabla 1}

Rendimientos promedio anuales IPC vs S\&P/BMV

\begin{tabular}{r|r|r|} 
Año & Ren IPC & \multicolumn{2}{r}{$\begin{array}{c}\text { Ren S\&P/BMV } \\
\text { México ESG Index }\end{array}$} \\
\hline $\mathbf{2 0 1 6}$ & $0.036 \%$ & $0.057 \%$ \\
\hline $\mathbf{2 0 1 7}$ & $0.033 \%$ & $0.037 \%$ \\
\hline $\mathbf{2 0 1 8}$ & $-0.062 \%$ & $-0.041 \%$ \\
\hline $\mathbf{2 0 1 9}$ & $0.021 \%$ & $0.050 \%$ \\
\hline $\mathbf{2 0 2 0}$ & $0.023 \%$ & $0.029 \%$ \\
\hline
\end{tabular}

Fuente: Elaboración propia.

Miranda Pegueros, M., López Castro, E. M., y Vega Zarate, C.
Vol. 7, núm. 19 / enero - abril del 2022

DOI: https://doi.org/10.36791/tcg.v7i19.136

Pp. 150-164 
Esto proporciona argumentos para discutir en futuras investigaciones, las cuales aborden una conceptualización de sostenibilidad basada en un proceso que debe de gestarse considerando tanto los ángulos sociales como empresariales, para en conjunto encontrar estrategias que encaminen a la sostenibilidad como un punto alcanzable para las próximas generaciones.

\section{Conclusiones}

El presente trabajo aborda un marco teórico que permite la construcción de premisas que integran los elementos de análisis que, desde la perspectiva empresarial, ayuden a una incorporación exitosa de estrategias de cuidado ambiental. Aceptar como ciertos los principios que se han propuesto, ayuda a la construcción teórica de futuros trabajos para comprender el comportamiento empresarial y sus motivaciones para el cambio en materia de sostenibilidad.

Por lo tanto, se entiende que el proceso mediante el cual las empresas abordan las problemáticas ambientales con motivación estratégica generará un beneficio en materia de ventaja competitiva con respecto a aquellas empresas que no logren adaptarse a los cambios y demandas de los grupos de presión y gobiernos. Lo anterior, no solo asegura que las empresas se comprometan de manera más activa para volverse sostenibles, sino que el intercambio de beneficios se comparten entre la empresa y la sociedad, obteniendo un juego donde ambas partes ganan.

Esta nueva conceptualización, permite una convergencia en los trabajos científicos cuyo sujeto de estudio se enfoca en la empresa. A través de lo anterior, se ha observado que la sostenibilidad no debe de estar peleada con la generación de riqueza, ya que este pensamiento desvía la gestión empresarial del objetivo principal que es mantener tanto a la empresa como a la sociedad.

En este caso la vigilancia en materia de flujo de información que se presenta por parte de las empresas deberá de ser de gran importancia para generar un flujo para los Stakeholders que permita un seguimiento oportuno, así como la elaboración de herramientas para evitar este tipo de comportamientos.

Finalmente, se espera mantener la apertura para nuevas líneas de investigación que ayuden a generar soluciones, sobre todo ante una problemática sobre el cuidado del medio ambiente y de sus repercusiones sociales, de tal forma que se genere una sinergia hacia una transición donde el cuidado medio ambiental no sea sinónimo de pérdidas bajo la óptica empresarial, al contrario, se relacione de manera estrecha con la maximización de beneficios económicos y por ende su impacto en el desarrollo económico y social.

\section{Referencias}

Adams, C. A., Potter, B., Singh, P. J., y York, J. (2016). Exploring the implications of integrated reporting for social investment (disclosures). The British Accounting Review, 48(3), 283-296. https://doi.org/10.1016/j.bar.2016.05.002

Adams, C. A., y Larrinaga, G. C. (2007). Engaging with organisations in pursuit of improved sustainability accounting and performance. Accounting, Auditing \& Accountability Journal, 20(1), pp. 333-355. https://doi.org/10.1108/09513570710748535

Agle, B., Donaldson, T., Freeman, R., Jensen, M., Mitchell, R., y Wood, D. (2008). Dialogue: Toward Superior Stakeholder Theory. Business Ethics Quarterly, 18. https://doi.org/10.5840/beq200818214 
Andrés, E. F., Salinas, E. M., y Vallejo, J. M. (2010). La influencia de factores de presión en el comportamiento medioambiental de la empresa: análisis del efecto moderador del tipo de actividad. Revista Europea de Dirección y Economía de la Empresa, 19(1), 127-146. https://dialnet.unirioja.es/servlet/articulo?codigo=3118 514.

Bebbington, J., Larrinaga, C., y Moneva, J. M. (2008). Corporate social reporting and reputation risk management. Accounting, Auditing \& Accountability Journal, 21(3), 337-361. https://doi.org/10.1108/09513570810863932

Blowfield, M., y Frynas, J. G. (2005). Setting New Agendas: Critical Perspectives on Corporate Social Responsibility in the Developing World. International Affairs (Royal Institute of International Affairs 1944-), 81(3), 499-513. http://www.jstor.org/stable/3569630.

Candelas, R. (2017). Hacia un Modelo Económico para el Desarrollo Sustentable. Centro de Estudios Sociales y de Opinión Pública (CESOP), documento de trabajo 259, México.

http://www5.diputados.gob.mx/index.php/camara/Cent ros-de-Estudio/CESOP/Novedades/Documento-detrabajo-Hacia-un-Modelo-Economico-para-elDesarrollo-Sustentable.

Carroll, A. (2009). A History of Corporate Social Responsibility: Concepts and Practices. In The Oxford Handbook of Corporate Social Responsibility (pp. 1946). https://doi.org/10.1093/oxfordhb/9780199211593.003. 0002

Carroll, A. B. (2015). Corporate social responsibility: The centerpiece of competing and complementary frameworks. Organizational Dynamics, 44, 87-96. http://dx.doi.org/10.1016/j.orgdyn.2015.02.002
Comisión Europea (2011). Estrategia renovada de la UE para 2011-2014 sobre la responsabilidad social de las empresas.

https://www.europarl.europa.eu/meetdocs/2009_2014/ documents/com/com_com(2011)0681_/com_com(2011 )0681_es.pdf.

Crane, A., McWilliams, A., Matten, D., Moon, J., y Siegel, D. (2009). The Corporate Social Responsibility Agenda. In The Oxford Handbook of Corporate Social Responsibility. https://doi.org/10.1093/oxfordhb/9780199211593.003. 0001

Delmas, M. A., Lyon, T. P., y Jackson, S. (2019). Using Market Forces for Social Good (2019). Chapter 15 in The Nonprofit Sector, A Research Handbook, Third Edition Edited by Walter Powell and Patricia Bromley, Stanford Press.

https://papers.ssrn.com/sol3/papers.cfm?abstract_id=34 57521.

Dyllick, T., y Muff, K. (2016). Clarifying the meaning of sustainable business: Introducing a typology from business-as-usual to true business sustainability. Organization \& Environment, 29(2), 156-174. https://doi.org/10.1177/1086026615575176

Earnhart, D., Khanna, M., y Lyon, T. (2014). Corporate Environmental Strategies in Emerging Economies. Review of Environmental Economics and Policy, 8, 164-185. https://doi.org/10.1093/reep/reu001

Eccles, R. G., Ioannou, I., y Serafeim, G. (2014). The impact of corporate sustainability on organizational processes and performance. Management Science, 60(11), 2835-2857.

https://doi.org/10.1287/mnsc.2014.1984
Miranda Pegueros, M., López Castro, E. M., y Vega Zarate, $\mathrm{C}$. 
Ergene, S., Banerjee, S. B., y Hoffman, A. J. (2020).

(Un) sustainability and organization studies: Towards a radical engagement. Organization Studies, 0170840620937892.

https://doi.org/10.1177/0170840620937892

Fernández, J., y Bajo, A. (2012). La Teoría del Stakeholder o de los Grupos de Interés, pieza clave de la RSE, del éxito empresarial y de la sostenibilidad.

ADResearch ESIC International Journal of Communication Research, 6, 130-143. https://dialnet.unirioja.es/servlet/articulo?codigo $=3980$ 299

Freeman, R. E. (2010). Strategic management: A stakeholder approach. Cambridge university press.

Friedman, M. (2007). The social responsibility of business is to increase its profits. In Corporate ethics and corporate governance. Springer, Berlin, Heidelberg, 173-178.

Gao, J., y Bansal, P. (2013). Instrumental and integrative logics in business sustainability. Journal of Business Ethics, 112(2), 241-255. https://doi.org/10.1007/s10551-012-1245-2

Hahn, T., Pinkse, J., Preuss, L., y Figge, F. (2015). Tensions in corporate sustainability: Towards an integrative framework. Journal of Business Ethics, 127(2), 297-316. https://doi.org/10.1007/s10551-0142047-5

Hang, M., Geyer-Klingeberg, J., Rathgeber, A., y Stöckl, S. (2018). Economic development matters: A meta-regression analysis on the relation between environmental management and financial performance. Journal of Industrial Ecology, 22(4), 720-744.
Husted, B. W., y Salazar, J. de J. (2005). Un estudio exploratorio sobre la estrategia social de empresas grandes ubicadas en México. Contaduría y Administración, (215), 9-23. https://www.redalyc.org/articulo.oa?id=39521502.

Kuhn, T., y Deetz, S. (2009). Critical Theory and Corporate Social Responsibility : Can/Should We Get Beyond Cynical Reasoning? The Oxford Handbook of Corporate Social Responsibility. https://doi.org/10.1093/oxfordhb/9780199211593.003. 0008

Lyon, T., y Maxwell, J. (2007). Corporate Social Responsibility and the Environment: A Theoretical Perspective. Review of Environmental Economics and Policy, 2, 240-260. https://doi.org/10.2139/ssrn.1011793

Mayo, A., Pérez, B. y Morales, Y. (2018). La sustentabilidad en el mercado de valores. Desarrollo regional sustentable y turismo. Universidad Nacional Autónoma de México y Asociación Mexicana de Ciencias para el Desarrollo Regional A.C., Coeditores. http://ru.iiec.unam.mx/3798/

Melé, D. (2009). Corporate Social Responsibility Theories. In The Oxford Handbook of Corporate Social Responsibility. https://doi.org/10.1093/oxfordhb/9780199211593.003. 0003

Miranda, M., y López, E. M. (2020). La Sustentabilidad en los Rendimientos Financieros de los Corporativos del Sector Industrial de las Empresas que Cotizan en la BMV. Revista de Gestión Empresarial y Sustentabilidad, 6(1), 64-75. https://148.216.29.53/index.php/rges/article/view/62 
Molina, E. C., Córdova, J. D., Meza, E. Z., y López, P. P. (2017). La responsabilidad social empresarial en las empresas del Ecuador. Ingeniería Industrial. Actualidad y Nuevas Tendencias, 5(18), 23-44. https://www.redalyc.org/pdf/2150/215052403003.pdf

Morales-Castro, J. A. (2015). La acreditación de sustentabilidad y su impacto en los márgenes de rentabilidad del sector industrial de la Bolsa Mexicana de Valores. Respuestas, 20(1), 16-29.

https://doi.org/10.22463/0122820X.256

Narayanan, V., y Adams, C. A. (2017). Transformative change towards sustainability: the interaction between organisational discourses and organisational practices. Accounting and Business Research, 47(3), 344-368. https://doi.org/10.1080/00014788.2016.1257930

ONU. (2015). Transformar nuestro mundo: la Agenda 2030 para el Desarrollo Sostenible. Asamblea General. http://www.un.org/ga/search/view_doc.asp?symbol=A/ 70/L.1\&Lang $=S$

Owen, D. L., y O’Dwyer, B. (2009). Corporate Social Responsibility: The Reporting and Assurance Dimension. In The Oxford Handbook of Corporate Social Responsibility. https://doi.org/10.1093/oxfordhb/9780199211593.003. 0017
Pérez, B. I. (2018). Evaluación financiera del rendimiento y el riesgo del IPC y el IPC sustentable de la bolsa mexicana de valores de 2012 a 2016 (Tesis de doctorado, Universidad Autónoma de Aguascalientes). http://bdigital.dgse.uaa.mx:8080/xmlui/handle/11317/1 573

Plasencia Soler, J. A., Marrero Delgado, F., Bajo Sanjuán, A. M., y Nicado García, M. (2018). Modelos para evaluar la sostenibilidad de las organizaciones. Estudios Gerenciales, 34(146), 63-73. https://doi.org/10.18046/j.estger.2018.146.2662

Rotner, E. (2016). Corporate Environmentalism: How Green is it Actually? Environmental Studies Honors Papers.

https://digitalcommons.conncoll.edu/envirohp/14/

Salazar, J., y Husted, B. W. (2009). Principals and Agents: Further Thoughts on the Friedmanite Critique of Corporate Social Responsibility. In The Oxford Handbook of Corporate Social Responsibility. https://doi.org/10.1093/oxfordhb/9780199211593.003. 0006

Savitz, A. W., y Weber, K. (2007). The sustainability sweet spot. Environmental Quality Management, 17(2), 17-28. https://doi.org/10.1002/tqem.20161 